\title{
G sonatem \\ Buffering Effect of in-patient Psychiatric Care on the Link Between Fear of Covid-19 and Mental Health Consequences
}

\section{Yuval Bloch ( $\nabla$ yuvalbloch10@gmail.com )}

Shalvata Mental Health Center, affiliated to Tel Aviv University Sackler Faculty of Medicine, Israel Sharon Shemesh

Shalvata Mental Health Center, affiliated to Tel Aviv University Sackler Faculty of Medicine, Israel

\section{Ariella Grossman-Giron}

Ariel University, Department of Behavioral Sciences.

\section{Hagai MaOz}

Shalvata Mental Health Center, affiliated to Tel Aviv University Sackler Faculty of Medicine, Israel

\section{Erica Cohenmehr}

Shalvata Mental Health Center, affiliated to Tel Aviv University Sackler Faculty of Medicine, Israel

\section{Libi Hertzberg}

Shalvata Mental Health Center, affiliated to Tel Aviv University Sackler Faculty of Medicine, Israel

\section{Uri Nitzan}

Shalvata Mental Health Center, affiliated to Tel Aviv University Sackler Faculty of Medicine, Israel

\section{Dana Tsur-Bitan}

Ariel University, Department of Behavioral Sciences.

\section{Research Article}

Keywords: Psychiatric admissions during the covid-19, lockdown, COVID-19, Psychological well-being scale (PWB)

Posted Date: March 3rd, 2021

DOl: https://doi.org/10.21203/rs.3.rs-250770/v1

License: (c) (i) This work is licensed under a Creative Commons Attribution 4.0 International License. Read Full License

Version of Record: A version of this preprint was published at Psychiatry Research Communications on February 1st, 2022. See the published version at https://doi.org/10.1016/j.psycom.2022.100027. 


\section{Abstract}

\section{Background\& Aimes}

Psychiatric admissions during the covid-19 pandemic were limited ignoring their possible benefit. The study focused on assessing the effect of the fear of covid on the mental health and well-being of inpatients as opposed to outpatients

\section{Methods}

During the first lockdown, forty-four in-patients and day care patients (in-patient group) and 74 outpatients (out-patient group) were recruited after an informed consent procedure. Fear from the infection was assed using the Fear of COVID-19 (FCV-19S), severity of mental health symptomatology was evaluated with the outcome questionnaire-45 (OQ-45)., wellbeing was assessed with the Psychological well-being scale (PWB).

\section{Outcomes}

There was no difference between the in-patient group and out-patient group in their fear of COVID-19 levels.

FCV-19 predicted changes in the outpatient $\mathrm{OQ}$ total score $(B=2.21, p<0.001), \mathrm{OQ}$ interpersonal relation subscale $(B=0.34, p=0.01)$, PWB total score $(B=-0.05, p<0.001)$, PWB environmental mastery subscale $(B=-.07, p<0.001)$ and PWB positive relation subscale $(B=-0.05, p<0.001)$, but not in the inpatient group.

\section{Conclusions}

Mental health and well-being of the out-patient group that had less therapeutic contact - unlike the inpatient group-correlated with the fear of covid. Supporting the hypothesis that intensive psychiatric therapy had a protective effect from the mental health consequences of "fear of covid".

\section{Introduction}

Mental health of psychiatric patients during the pandemic

The current Coronavirus-19 (covid-19) pandemic is an unprecedented global crisis, affecting the mental health and wellbeing of the general population worldwide (1). The combination of the "fear of covid-19 and its' related consequences, such as the demand for social distancing, are recognized as significant stressors for everyone (1). Researchers have argued that patients in need of psychiatric care prior to the pandemic raise special concerns (2). The literature from past disasters and catastrophes indicates an unpredictable response may be expected of severely mentally ill patients. For example, one week after a high magnitude tsunami in Japan on March 11, 2011 and a subsequent explosion at the Fukushima Daiichi nuclear power plant, researchers found that there was no symptomatic change in the condition of 
two thirds of the psychiatric inpatients, and there were even some patients with improvement(3). Nonetheless, preliminary evidence from the current pandemic indicates that the fear of covid-19 among patients with previous mental health disorders, may be at least as severe as in the general population(35). Many people with mental health difficulties share the suggested risk factors found in the general population for having a higher mental health risk, including: lower socioeconomic condition, having less coping skills and less social support, and having higher measures of hostility towards others (6). Psychiatric care is considered imperative in treating people with mental illness, especially in times of crisis. In the pre-covid-19 era, routine psychiatric care was largely based on direct social contacts (7).

Social contact in psychiatric care

Direct social contacts are relevant to all forms of psychiatric care, including psychiatric evaluations, psychiatric follow-ups and psychotherapy. Previously, there was only very limited use of tele- psychiatry, and direct personal contact was considered by many a hallmark of psychiatric therapy (2). Inpatient wards and day hospitalizations are also based on close social interactions. They include recurring meetings with different staff members, group sessions, and a "therapeutic socially active environment", characterized by shared meals and numerous informal interactions (7).

Risks and benefits during a pandemic

However, findings have shown that this proximity in psychiatric hospitalizations poses a risk for spreading the infection (8). Reports of in-word contamination and consequent fatalities have been published worldwide (9). Thus, as the pandemic progresses, reports indicate a decline in psychiatric admissions and inpatient care, while patients are discharged as early as possible and the decision to stay in intensive psychiatric care is based on the level of immediate risk to self or others $(10,11)$. Previous researchers have shared their experience and knowledge of caring for patients in this context, aiming at minimizing the spread of infection under these circumstances $(7,8,12)$. In order to guide clinicians and policy makers about the multifaceted consequences of psychiatric hospitalization during the pandemic, it seems crucial to assess not only the risks, but also the potential mental health benefits. To quantitate gains of complex interventions such as psychiatric hospitalization or day-hospitalization is an extremely challenging task, as it necessitates the use of an accepted outcome measure, in naturalistic settings, whilst partaking in numerous trans diagnostic interventions, with various possible biological and psychosocial confounding variables. However, the task of elucidating the effect of inpatient psychiatry in the context of the current pandemic is essential, and many questions remain unanswered. For example, thus far, it is not clear if the more severely ill patients that required hospitalization during the pandemic suffer from "fear of covid-19" to the same extent as less severely ill patients who require only outpatient therapy.

The current study

In the current study, we aimed at assessing fear of COVID-19, symptomatic distress levels, as well as wellbeing among inpatients and outpatients, during the first lockdown, in Shalvata Mental Health Center 
in Israel. Based on recent literature, showing that the fear of covid-19 among psychiatric patients is as severe as in the general population (3-5), we hypothesized that both groups would exhibit similar levels of "fear of covid-19". Moreover, seeing that the fear itself has been shown to have detrimental effects on symptomatology and wellbeing of different patient groups $(3,4,13)$, our second hypothesis was that inpatients- would be unaffected from the determinate effects of the fear of covid-19 on mental health and wellbeing. This presumed protective effect would emerge beyond the effect of possible differences between the groups studied such as symptomatic severity and the presence of psychosis.

\section{Methods}

\section{Procedure and Participants}

The study was approved by the Shalvata Mental Health Center institutional review board (IRB, approval number: 007-20-SHA). All research was performed in accordance with relevant guidelines/regulations.

All participants were recruited between April 26th and June 6th of 2020, a short time after a general COVID-19 pandemic lockdown was mandated in Israel. The regulations had a profound effect on mental health services. They included discharging patients to less intense therapy when possible, limiting guest visits and holidays in inpatient wards, and avoiding frontal meetings for outpatients when possible (including rescheduling appointments and moving to tele psychiatry). Study recruitment concluded at the beginning of June because at that point, many of the strict regulations had been lifted, and with that, the atmosphere surrounding COVID had shifted. Inclusion criteria for inpatient group consisted of being an inpatient or a day patient and being able to cooperate in the questionnaire assessment. Inclusion criteria for the outpatient group was being an outpatient at the time of the study with consultations occurring up to weekly (usually less).

We recruited participants by distributing publications to both staff and patients in the different units in Shalvata Mental Health Center.

Forty-four participants were recruited for the inpatient and day patient group. They were recruited from 3 inpatient wards and one outpatient ward. There was a total of 217 patients in the 4 units during the relevant time. Participants who had agreed to take part in the study were asked to sign informed consent and to fill out a hard copy of the study questionnaires. For simplicity, this will be referred to as the inpatient group (including both in-patients and day patients).

Seventy-four outpatients were recruited for the outpatient group. Beyond written communication with outpatients, 120 patients participating in a former study assessing the effect of process and outcome feedback in distressed outpatients were contacted again for recruitment to the current study. After signing an online informed consent, outpatient respondents performed an online version of the same survey given to the inpatient participants using an online data gathering software (Qualtrics).

The mean age of the 128 patients was $36.5(S D=13.76) .60 .2 \%$ were men and $39.8 \%$ were women. 
Measures

The Outcome Questionnaire-45 (OQ-45). A commonly used, well-validated and reliable self-report questionnaire. It is used to assess patient outcomes over the course of psychotherapy. This measure aims at a comprehensive trans-diagnostic assessment of the patients' clinical condition. It consists of 45 items, evaluating three different dimensions: (a) symptom distress, (b) interpersonal relationships, and (c) social role performance. While the total score range is $0-180$, the cutoff score between clinical and nonclinical populations is $63(14,15)$.

Psychological well-being scale (PWB). A self-report questionnaire created for the purpose of evaluating six elements of psychological well-being: personal growth, purpose in life, self-acceptance, environmental mastery, positive relations with others, and autonomy. Answers are given on a 6-point Likert scale ranging from 1 (strongly disagree) to 6 (strongly agree). A mean score is calculated for each dimension of wellbeing, with higher scores indicating higher well-being in all domains. Previous studies among psychiatric outpatients have shown that this scale is sensitive to changes in well-being. For the purpose of the current study, we utilized the total score of the PWB, as well as the personal growth, purpose-in-life, and self-acceptance factors. The alpha coefficient of the PWB in the current sample indicated high internal reliability (Cronbach's alpha $=.95)(16)$.

Fear of COVID-19 (FCV-19S). A self-report scale designed to measure fear of COVID-19 (17). The questionnaire consists of 7 items describing pandemic-related emotional fear reactions. Items are rated on a five-item Likert-type scale ranging from 1 (strongly disagree) to 5 (strongly agree) and a total sum score is calculated. The total scale range is 7-35, with higher scores demonstrating higher fear of COVID19. This scale recently showed good psychometric properties in an Israeli sample (18). The alpha coefficient of the FCV-19S in the current sample indicated high internal reliability (Cronbach's alpha = $.91)$.

Statistical Analyses

Demographic and clinical differences between the inpatient and outpatient groups were assessed using a chi square test for categorical variables (sex) and t-test for continuous variables (table 1\&2).

In order to assess the moderating effect of being an inpatient or outpatient on the association between fear of COVID-19 (FCV) and clinical severity and psychological well-being (as represented in the 0Q-45 and PWB subscales), we first performed test of normality on all outcome measures. The Hayes process script was then used to assess the moderating effect of the group on the predictive effect of fear of COVID-19 on the OQ-45 and PWB total scores and subscales (19). To substantiate our findings, we controlled for covariates that differed between the two groups. All statistical procedures were conducted using SPSS version 25 .

\section{Results}


Comparison of the two patient groups

The study groups were compared to evaluate differences in possible confounders. Significant differences were found in gender, marital status, education, and diagnosis (nonpsychotic or psychotic, including schizophrenia bipolar and psychotic depression). (Table 1)

Table 1. Demographic characteristics of the sample 


\begin{tabular}{|c|c|c|c|c|c|}
\hline \multicolumn{2}{|l|}{ Variable } & $\begin{array}{l}\text { Inpatients } \\
(\mathrm{n}=44)\end{array}$ & $\begin{array}{l}\text { Outpatients } \\
(\mathrm{n}=73)\end{array}$ & $\begin{array}{l}\text { Total } \\
(\mathrm{N}=117)\end{array}$ & $p$ \\
\hline \multicolumn{2}{|c|}{ Sex (Males; Value \%) } & $21(47.7 \%)$ & $49(67.1 \%)$ & 70 (59.8\%) & $p<0.05$ \\
\hline \multicolumn{2}{|l|}{ Age (Mean; SD) } & $34.27(13.03)$ & $37.80(14.11)$ & $\begin{array}{l}36.51 \\
(13.83)\end{array}$ & N.S. \\
\hline \multicolumn{2}{|c|}{ Birth Country (Israel) } & $36(81.8 \%)$ & $56(76.7 \%)$ & $92(78.6 \%)$ & N.S. \\
\hline \multirow[t]{5}{*}{$\begin{array}{l}\text { Socio-economic } \\
\text { status }\end{array}$} & $\begin{array}{l}\text { Way below } \\
\text { average }\end{array}$ & $18(40.9 \%)$ & $24(32.4 \%)$ & $42(35.9 \%)$ & \multirow[t]{5}{*}{ N.S. } \\
\hline & Below average & $10(22.7 \%)$ & $20(27 \%)$ & $30(25.6 \%)$ & \\
\hline & Average & $11(25 \%)$ & $16(21.6 \%)$ & $27(23.1 \%)$ & \\
\hline & $\begin{array}{l}\text { Higher than } \\
\text { average }\end{array}$ & $3(6.8 \%)$ & $11(15.1 \%)$ & $14(12 \%)$ & \\
\hline & $\begin{array}{l}\text { Way higher than } \\
\text { aver }\end{array}$ & $2(4.5 \%)$ & $2(2.7 \%)$ & $4(3.4 \%)$ & \\
\hline \multirow[t]{6}{*}{ Marital Status } & Single & $32(72.7 \%)$ & $24(32.4 \%)$ & $56(47.9 \%)$ & \multirow[t]{6}{*}{$p<0.001$} \\
\hline & Married & $4(9.1 \%)$ & $34(46.6 \%)$ & 38 (32.5\%) & \\
\hline & Partnered & $2(4.5 \%)$ & $9(12.2 \%)$ & $11(9.4 \%)$ & \\
\hline & Divorced & $5(11.4 \%)$ & $5(6.8 \%)$ & $10(8.5 \%)$ & \\
\hline & Separated & $1(2.3 \%)$ & - & $1(0.9 \%)$ & \\
\hline & Widow & - & $1(1.4 \%)$ & $1(0.9 \%)$ & \\
\hline \multirow[t]{5}{*}{ Education } & $\begin{array}{l}\text { Elementary } \\
\text { School }\end{array}$ & $4(9.1 \%)$ & $2(2.7 \%)$ & $6(5.1 \%)$ & \multirow[t]{5}{*}{$p<0.05$} \\
\hline & High School & $30(68.2 \%)$ & $30(41.1 \%)$ & $60(51.3 \%)$ & \\
\hline & BA & $9(20.5 \%)$ & $29(39.2 \%)$ & $38(32.5 \%)$ & \\
\hline & MA & $1(2.3 \%)$ & $11(14.9 \%)$ & $12(10.3 \%)$ & \\
\hline & Phd & - & $1(1.4 \%)$ & $1(0.9 \%)$ & \\
\hline \multicolumn{2}{|c|}{$\begin{array}{l}\text { Psychotic Spectrum Diagnosis (Yes; } \\
\text { Value \%) }\end{array}$} & 15 (36.6\%) & $3(4.1 \%)$ & $18(15.8 \%)$ & $p<0.001$ \\
\hline
\end{tabular}

There was no difference in fear of COVID-19 between the inpatient group and outpatient group.

The groups differed in OQ-45 total score as well as in all its three subscales: symptom distress, interpersonal relations, and social role, indicating that the in-patient group reported generally higher distress levels $(t(114)=3.28, p \leq 0.05)$, more subjective discomfort (symptom distress subscale; $t(114)=3.31, p<0.01)$, more loneliness and involvement in interpersonal conflicts (interpersonal relation 
subscale: $\mathrm{t}(114)=2.05, \mathrm{p}<0.05)$ and more difficulties in completing their duties (social role subscale: $t(114)=2.83, p \leq 0.05)$ compared to their counterparts in the low therapy intensity group.

With regard to the psychological well-being, as expected, an increasing degree of well-being $(\mathrm{t}(110)=-2.55$, $p<0.05)$, as well as a sense of environmental mastery $(t(110)=3.85, p<0.001)$, positive relation $(t(108)=-2.35, p<0.05)$, and self-acceptance $(t(107)=-2.52, p=0.01)$ were observed in the outpatient group compared to the in-patient group (Table 2).

Table 2. Between-group differences of the study variable

\begin{tabular}{|llll|}
\hline Variable & Inpatients $(\mathrm{n}=44)$ & Outpatients $(\mathrm{n}=\mathbf{7 3})$ & $\boldsymbol{P}$ \\
\hline Fear of COVID-19 scale & $15.25(7.61)$ & $15.72(6.55)$ & 0.73 \\
\hline OQ-45 Total mean** & $89.61(29.59)$ & $71.15(29.89)$ & 0.001 \\
\hline OQ-45 Symptom Distress** & $54.32(19.47)$ & $42.18(18.96)$ & 0.001 \\
\hline OQ-45 Interpersonal Relations** & $20.07(7.47)$ & $17.12(7.52)$ & 0.04 \\
\hline OQ-45 Social Role** & $15.23(6.69)$ & $11.85(5.93)$ & 0.005 \\
\hline PWB Total mean** & $3.45(0.71)$ & $3.84(0.80)$ & 0.12 \\
\hline PWB Autonomy & $3.77(0.82)$ & $3.92(0.84)$ & 0.34 \\
\hline PWB Environmental mastery *** & $2.76(1.02)$ & $3.57(1.12)$ & $<0.001$ \\
\hline PWB Personal Growth & $4.10(0.89)$ & $4.18(0.80)$ & 0.63 \\
\hline PWB Positive Relation** & $3.54(0.89)$ & $4.01(1.08)$ & 0.02 \\
\hline PWB Purpose in Life & $3.63(0.86)$ & $3.89(0.91)$ & 0.14 \\
\hline PWB Self-Acceptance** & $2.92(1.16)$ & $3.51(1.20)$ & 0.01 \\
\hline
\end{tabular}

${ }^{*}=p<0.1 ;{ }^{* *}=p<0.05 ;{ }^{* *}=p<0.001 ; P W B=$ Psychological Well Being; OQ=Outcome Questionnaire.

Moderation Analysis

The Shapiro-Wilk test of normality indicated a normal distribution of all process and outcome variables. Being an in-or outpatient had a moderating effect on the impact of FCV on the OQ total score $(B=1.49$, $t=2.14,95 \% \mathrm{Cl}: 0.11 ; 2.87, p=0.03), \mathrm{OQ}$ interpersonal relation subscale $(B=0.45, t=2.28,95 \% \mathrm{Cl}: 0.06 ; 0.85$, $p=0.02)$, PWB total score $(B=-0.04, t=-2.08,95 \% \mathrm{Cl}:-0.08 ; 0.00, p=0.04)$, PWB environmental mastery subscale $(B=-0.06, t=-2.25,95 \% \mathrm{Cl}:-0.11 ;-0.01, p=0.03)$ and $P W B$ positive relation subscale $(B=-0.07$, $\mathrm{t}=-2.68,95 \% \mathrm{Cl}:-0.13 ;-0.02, \mathrm{p}=0.01$ ) (Table 3 ).

Table 3 Moderation analyses for the effect of intensive therapy on the association between fear of COVID-19 and psychological well-being and distress 


\begin{tabular}{|c|c|c|c|c|c|c|}
\hline & & Effect & SE & $T$ & $95 \% \mathrm{Cl}$ & $P$ \\
\hline \multirow[t]{2}{*}{ 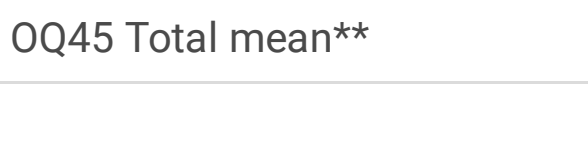 } & Inpatient & 0.63 & 0.53 & 1.20 & $-0.41 ; 1.67$ & 0.23 \\
\hline & Outpatient & 2.12 & 0.46 & 4.63 & $1.21 ; 3.03$ & $<0.001$ \\
\hline \multirow[t]{2}{*}{ OQ symptom distress* } & Inpatient & 0.59 & 0.33 & 1.77 & $-0.07 ; 1.24$ & 0.08 \\
\hline & Outpatient & 1.42 & 0.29 & 4.92 & $0.85 ; 1.99$ & $<0.001$ \\
\hline \multirow[t]{2}{*}{ OQ social role } & Inpatient & 0.16 & 0.12 & 1.29 & $-0.08 ; 0.39$ & 0.20 \\
\hline & Outpatient & 0.36 & 0.11 & 3.40 & $0.15 ; 0.57$ & $<0.001$ \\
\hline \multirow[t]{2}{*}{ OQ interpersonal relations ${ }^{\star \star}$} & Inpatient & -0.11 & 0.15 & -0.74 & $-0.41 ; 0.19$ & 0.46 \\
\hline & Outpatient & 0.34 & 0.13 & 2.62 & $0.08 ; 0.60$ & 0.01 \\
\hline \multirow[t]{2}{*}{ PWB Total mean ${ }^{\star \star}$} & Inpatient & -0.01 & 0.01 & -0.39 & $-0.03 ; 0.02$ & 0.70 \\
\hline & Outpatient & -0.05 & 0.01 & -3.61 & $-0.07 ;-0.02$ & $<0.001$ \\
\hline \multirow[t]{2}{*}{ PWB Autonomy } & Inpatient & -0.04 & 0.02 & -2.65 & $-0.08 ;-0.01$ & 0.01 \\
\hline & Outpatient & -0.04 & 0.01 & -2.76 & $-0.07 ;-0.01$ & 0.01 \\
\hline \multirow[t]{2}{*}{ PWB Environmental mastery** } & Inpatient & 0.00 & 0.02 & -0.22 & $-0.04 ; 0.03$ & 0.83 \\
\hline & Outpatient & -0.07 & 0.02 & -3.67 & $-0.10 ;-0.03$ & $<0.001$ \\
\hline \multirow[t]{2}{*}{ PWB Personal Growth } & Inpatient & 0.00 & 0.02 & 0.21 & $-0.03 ; 0.04$ & 0.84 \\
\hline & Outpatient & -0.02 & 0.02 & -1.44 & $-0.05 ; 0.01$ & 0.15 \\
\hline \multirow[t]{2}{*}{ PWB Positive Relation** } & Inpatient & 0.02 & 0.02 & 0.91 & $-0.2 ; 0.06$ & 0.37 \\
\hline & Outpatient & -0.05 & 0.02 & -3.02 & $-0.09 ;-0.02$ & $<0.001$ \\
\hline \multirow[t]{2}{*}{ PWB Purpose in Life } & Inpatient & -0.01 & 0.02 & -0.63 & $-0.04 ; 0.02$ & 0.53 \\
\hline & Outpatient & -0.04 & 0.02 & -2.62 & $-0.07 ;-0.01$ & $<0.001$ \\
\hline \multirow[t]{2}{*}{ PWB Self-Acceptance } & Inpatient & -0.01 & 0.02 & -0.40 & $-0.06 ; 0.04$ & 0.69 \\
\hline & Outpatient & -0.04 & 0.02 & -2.18 & $-0.08 ; 0.00$ & 0.03 \\
\hline
\end{tabular}

Simple slope regression analysis indicated that FCV-19 predicted changes in the outpatient $\mathrm{OQ}$ total score $(B=2.21, p<0.001), O Q$ interpersonal relation subscale $(B=0.34, p=0.01)$, PWB total score $(B=-0.05$, $p<0.001), P W B$ environmental mastery subscale $(B=-.07, p<0.001)$ and $P W B$ positive relation subscale $(B=-0.05, p<0.001)$, but not in the inpatient group (fig 1). 


\section{Discussion}

The central finding of the present study is that unlike in the inpatient group, the mental condition and well-being of the outpatient group, which have less psychiatric contact, correlates with the fear of covid19. This supports our hypothesis that intensive psychiatric care has a protective effect on the consequences of "fear of covid-19" on mental health and well-being.

While the groups differ in mental health and wellbeing, they do not differ in their level of fear of the pandemic. As expected, the reaction to this significant stressor is fear. To an extent, "fear of covid-19" is a "normative response" reported in the general population-not only in both study patient groups, but also in the general population in health professionals and different patient groups across countries $(1,20,21)$.

Previous studies have focused on the prevalence of mental health symptoms during the pandemic in different groups $(3,4,22)$. In this respect, it appears that patients who have previous mental health difficulties are more vulnerable to the mental health consequences of the "fear of covid-19" (3-5).

These findings point to the current condition, but they cannot help in evaluating the causes of the mental health difficulties. While some arise from the mental burden posed by the fear itself, difficulties related to the current economic circumstances or the damage to physical health, are indistinguishable. A more precise understanding of the course of mental health deterioration is important because if the reasons are related directly to economic circumstances, they will probably demand different interventions. Some studies focused on evaluating the "fear of covid-19" itself $(17,18)$. The presence of fear is important, as it helps us conceptualize the "fear of covid" as a stressor, but has limited immediate clinical relevance. As mental health professionals and researchers in the field, we are interested not just in the presence of the "fear of covid-19", or in the presence of mental health symptoms and effects on wellbeing, but further, in a better understanding of the connection between them. In the current study we aimed to understand the burden of the perceived "fear of covid" on mental health and wellbeing of our patients, and attempted to study the protective effect of our common intensive therapies: inpatient or day-care psychiatric admissions. With the limitations of a cross sectional study, we used the correlation between "fear of covid-19" and patients' mental health symptomatology and wellbeing. In this sense, our findings in the outpatient group of the current study support the connection between the "fear of covid-19" and both mental health and psychological wellbeing. This probably points to the "fear of covid-19" itself, or to the ability to cope with this fear as therapeutic hubs-that need to be tackled even during the pandemic.

In the setting of a pandemic, treating psychiatric patients, especially those who necessitate more intensive treatment, poses a challenge due to the risk of spreading the disease and endangering patients' physical health $(9,10,23)$. Based on these dangers, actual therapeutic encounters were generally minimized in the face of the pandemic, and psychiatric inpatient therapy, day therapy, and all forms of intensive therapy were extremely limited $(9,10)$. Thus, not surprisingly, in the current study the in-patients 
had more psychiatric symptomatology (as reflected by results of the $\mathrm{OQ}$ ) and worse well-being (as reflected by results of the PWB).

Our second aim was to study the protective effect of our common intensive therapies: inpatient or daycare psychiatric admissions on the effect of "fear of covid-19" on mental health and psychological wellbeing. Unlike the outpatients, mental health and psychological well-being of the inpatients did not correlate with the "fear of covid-19". This supports a protective effect of the inpatient setting from the mental health consequences of the "fear of covid-19". In accord with this line of thought, recent studies have aimed at linking risk and protective factors in assessing prospectively the mental health outcome of the pandemic $(6,24)$. Duan et al. studied the general population and conducted two evaluations, one at the peak, and a second at the remission of the covid-19 pandemic. They pointed to social support as a "buffer" or a resilient factor, protecting from the malicious cascade from stress to depression (6). The fact that there was no difference in the level of "fear of covid-19" between the in-patients and outpatients in the current study can support this "buffer hypothesis". Duan et al. found that an increased level of perceived stress was a risk factor for worse outcome, and the use of negative coping strategies played a potential intermediating role in the deterioration. In a longitudinal assessment of participants from the community, Schafer et al. demonstrated that low "sense of coherence" before the pandemic was a risk factor for the development of mental health symptoms during the pandemic (24). Using other evaluation tools (OQ-45) the in-patient group in the current study was found to have higher distress levels, more subjective discomfort, more loneliness and involvement in interpersonal conflicts, and (PWB) less sense of environmental mastery, less positive relation and less self-acceptance. All these would be considered risk factors for the effects of the fear- possibly stressing the importance of being in intensive psychiatric therapy as a protective factor for mental health consequences.

The decision to treat patients, especially when treatment entails close encounters with therapists and other patients as in the in-patient or day care setting, cannot be taken lightly in the throes of a pandemic. There are current reports on a decline in the use of psychiatric services referrals and admissions during the lockdown weeks at the beginning of the pandemic $(10,11)$. Psychiatrists and therapists during the pandemic focused on the physical dangers and possibly neglected possible mental health consequences. This is understandable because the field did not have data about possible benefits of therapy during these troubling times. Due to the risk of contamination, it is important to study and quantify mental health benefits of therapy in these unique circumstances.

In the current study, we were able to point to a possible protective effect of the in-patient and day-therapy on the immediate detrimental mental health effects and psychological well-being effects of the fear of Covid. The intensive inpatient setting could not protect from the fear itself, but our results support the ability to dissociate the fear from its dangerous mental health consequences at least during the first wave of the pandemic.

Study limitations include the methodology of a naturalistic study. There was no randomization between the study groups. It seems that a randomized controlled study would be considered unethical. Our 
analysis attempted to tackle this problem by examining the significant clinical differences between the two patient groups as co-variants in the moderation analysis. Another limitation is the small group size and the patients' heterogeneity in diagnosis and disease severity. This limitation can be tackled by future larger scale studies-and in this respect our findings are probably a call for the importance in conducting such large scale studies. Probably the most important limitation relates to the cross sectional immediate effects. Longitudinal studies will be able to evaluate if the in-patient and day therapy had a protective lasting effect from the mental health stress afflicted from the "fear of covid".

\section{Declarations}

\section{All Authors have made substantial contribution to the attached study and fulfilled the 4 required criteria:}

1. Substantial contributions to the conception or design of the work; B, S.S., GG. A., ,H.M., H.L., N.U.,TB. D. and or the acquisition of the data, Y.B., S.S., GG. A., H.M., E.C., H.L., N.U., TB. D., and or the analysis of the data Y.B., S.S., GG. A., E.C., H.L., TB. D. and or interpretation of data for the work Y.B., S.S., GG. A., H.M., E.C., H.L., N.U., TB. D.

2. Drafting the work or revising it critically for important intellectual content; B. , S.S., GG. A., H.M., E.C., H.L., N.U., TB. D.

3. Final approval of the version to be published B, S.S., GG. A., H.M., E.C., H.L., N.U., TB. D.

4. Agreement to be accountable for all aspects of the work in ensuring that questions related to the accuracy or integrity of any part of the work are appropriately investigated and resolvedB. S.S., GG. A, H.M., E.C., H.L., N.U., TB. D.

Conflict of interests: None to declare.

\section{References}

1. Salari N, Hosseinian-Far A, Jalali R, Vaisi-Raygani A, Rasoulpoor S, Mohammadi M, et al. Prevalence of stress, anxiety, depression among the general population during the COVID-19 pandemic: a systematic review and meta-analysis. Global Health. 2020;16(1):57.

2. Bojdani E, Rajagopalan A, Chen A, Gearin P, Olcott W, Shankar V, et al. COVID-19 Pandemic: Impact on psychiatric care in the United States. Psychiatry Res. 2020;289:113069.

3. Liu CH, Stevens C, Conrad RC, Hahm HC. Evidence for elevated psychiatric distress, poor sleep, and quality of life concerns during the COVID-19 pandemic among U.S. young adults with suspected and reported psychiatric diagnoses. Psychiatry Res. 2020;292:113345.

4. Hao F, Tan W, Jiang L, Zhang L, Zhao X, Zou Y, et al. Do psychiatric patients experience more psychiatric symptoms during COVID-19 pandemic and lockdown? A case-control study with service and research implications for immunopsychiatry. Brain Behav Immun. 2020;87:100-6.

5. Gonzalez-Blanco L, Dal Santo F, Garcia-Alvarez L, de la Fuente-Tomas L, Moya Lacasa C, Paniagua G, et al. COVID-19 lockdown in people with severe mental disorders in Spain: Do they have a specific 
psychological reaction compared with other mental disorders and healthy controls? Schizophr Res. 2020.

6. Duan H, Yan L, Ding X, Gan Y, Kohn N, Wu J. Impact of the COVID-19 pandemic on mental health in the general Chinese population: Changes, predictors and psychosocial correlates. Psychiatry Res. 2020;293:113396.

7. Li L. Challenges and Priorities in Responding to COVID-19 in Inpatient Psychiatry. Psychiatr Serv. 2020;71(6):624-6.

8. Spitzer Sverd S, Gardner LE, Cabassa JA, Schneider M, Noone RH, Jahdi MH, et al. A Bronx tale: Exposure, containment and care on inpatient psychiatry units during COVID-19. Gen Hosp Psychiatry. 2020.

9. Barnett B, Esper F, Foster CB. Keeping the wolf at bay: Infection prevention and control measures for inpatient psychiatric facilities in the time of COVID-19. Gen Hosp Psychiatry. 2020;66:51-3.

10. Kolbaek P, Norremark B, Ostergaard SD. Forty Percent Reduction in Referrals to Psychiatric Services during the COVID-19 Pandemic. Psychother Psychosom. 2020:1-2.

11. Flevaud L, Pham A, Gourevitch R. [A sharp drop in psychiatric emergency admissions during lockdown]. Ann Med Psychol (Paris). 2020.

12. Du N, OuYang Y, Chen Y. The experience of prevention measures taken by the psychiatric hospital during the emergence of asymptomatic patients with COVID-19. Psychiatry Res. 2020;291:113109.

13. Davide P, Andrea P, Martina O, Andrea E, Davide D, Mario A. The impact of the COVID-19 pandemic on patients with OCD: Effects of contamination symptoms and remission state before the quarantine in a preliminary naturalistic study. Psychiatry Res. 2020;291:113213.

14. Gross R, Glasser S, Elisha D, Tishby O, Madar Jacobson D, Levitan G, et al. Validation of the Hebrew and Arabic Versions of the Outcome Questionnaire (OQ-45). Isr J Psychiatry Relat Sci. 2015;52(1):339.

15. Timman R, de Jong K, de Neve-Enthoven N. Cut-off Scores and Clinical Change Indices for the Dutch Outcome Questionnaire (OQ-45) in a Large Sample of Normal and Several Psychotherapeutic Populations. Clin Psychol Psychother. 2017;24(1):72-81.

16. Ryff CD, Keyes CL. The structure of psychological well-being revisited. J Pers Soc Psychol. 1995;69(4):719-27.

17. Ahorsu DK, Lin CY, Imani V, Saffari M, Griffiths MD, Pakpour AH. The Fear of COVID-19 Scale: Development and Initial Validation. Int J Ment Health Addict. 2020:1-9.

18. Tzur Bitan D, Grossman-Giron A, Bloch Y, Mayer Y, Shiffman N, Mendlovic S. Fear of COVID-19 scale: Psychometric characteristics, reliability and validity in the Israeli population. Psychiatry Res. 2020;289:113100.

19. Hayes AF. PROCESS: A Versatile Computational Tool for Observed Variable Mediation, Moderation, and Conditional Process Modeling. Columbus, $\mathrm{OH}$ : The Ohio State University. . Available at: http://wwwafhayescom/public/process2012pdf. 2012. 
20. Zhang XR, Huang QM, Wang XM, Cheng X, Li ZH, Wang ZH, et al. Prevalence of anxiety and depression symptoms, and association with epidemic-related factors during the epidemic period of COVID-19 among 123,768 workers in China: A large cross-sectional study. J Affect Disord. 2020;277:495-502.

21. Campion J, Javed A, Sartorius N, Marmot M. Addressing the public mental health challenge of COVID-19. Lancet Psychiatry. 2020;7(8):657-9.

22. Mazza C, Ricci E, Biondi S, Colasanti M, Ferracuti S, Napoli C, et al. A Nationwide Survey of Psychological Distress among Italian People during the COVID-19 Pandemic: Immediate Psychological Responses and Associated Factors. Int J Environ Res Public Health. 2020;17(9).

23. Zhang E, LeQuesne E, Fichtel K, Ginsberg D, Frankle WG. In-patient psychiatry management of COVID-19: rates of asymptomatic infection and on-unit transmission. BJPsych Open. 2020;6(5):e99.

24. Schafer SK, Sopp MR, Schanz CG, Staginnus M, Goritz AS, Michael T. Impact of COVID-19 on Public Mental Health and the Buffering Effect of a Sense of Coherence. Psychother Psychosom. 2020:1-7.

\section{Figures}
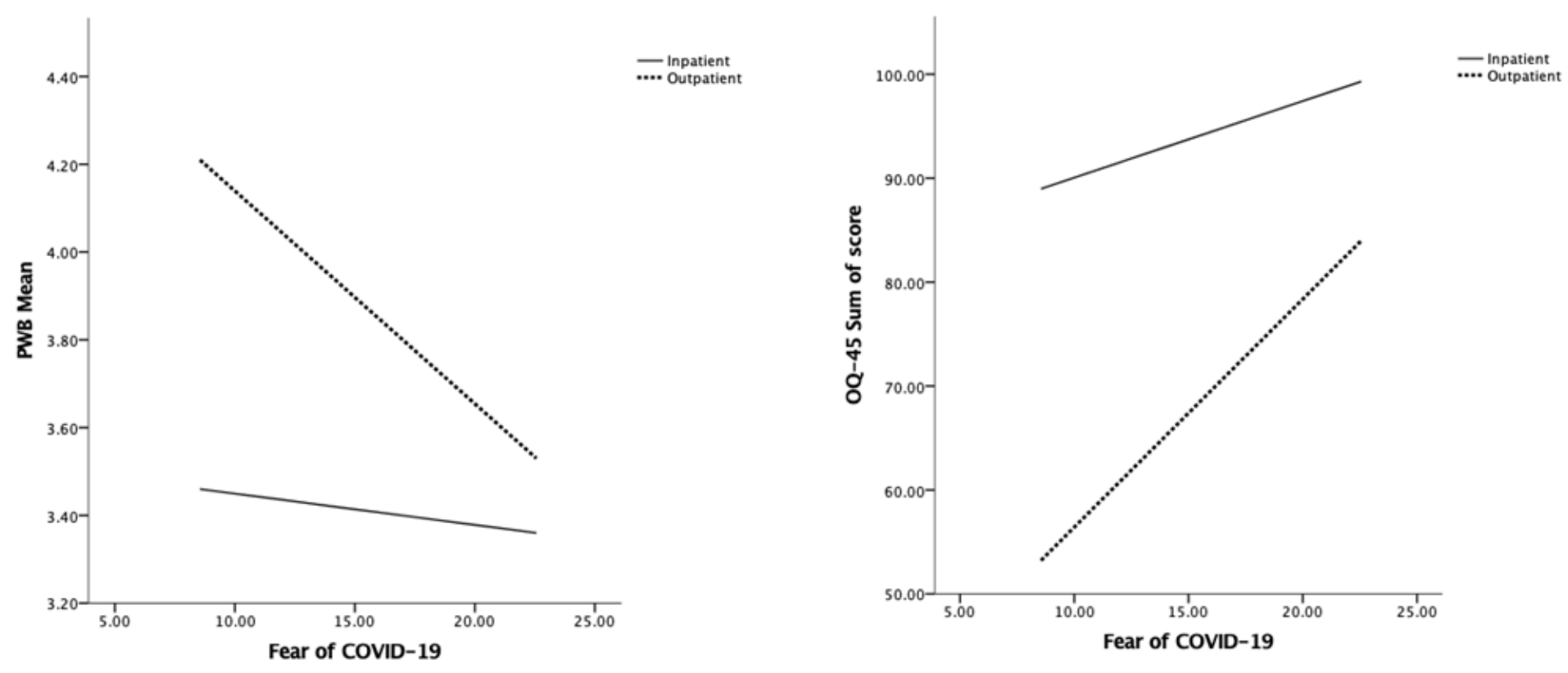

\section{Figure 1}

Simple slope regression analysis for the prediction of clinical state (OQ-45) and well-being (PWB) by fear of covid (FCV-19) in out-patients as opposed to in-patients. 